\title{
Journey of Gliomas- from Morphology to Genetics
}

\author{
Debajyoti Chatterjee, MD, DM
}

Assistant professor, Department of Histopathology,

Postgraduate Institute of Medical Education and Research (PGIMER), Chandigarh, India.

\begin{abstract}
Gliomas are the commonest brain tumors, both in adults and children. In the past one decade there is a massive development in the understanding in the pathogenesis and genetics of glial tumors. With the incorporation of the molecular information, gliomas are now classified based both on their morphology and genetics. These changes have been adapted in the current (2016) world health organization (WHO) revised classification of tumors of central nervous system. Incorporation of the molecular information has helped in the accurate classification of glial tumors, which provides important prognostic and predictive information. In this review article, we shall have a brief look at the morphology of the glial tumors with their molecular pathology, in the light of the current literature.
\end{abstract}

Keywords: Glioma, glioblastoma, diffuse midline glioma, isocitrate dehydrogenase, TERTpromoter

\section{Manuscript}

Glioma is the most common brain tumor among all the age groups. Gliomas were classified traditionally based on their histology as astrocytoma, oligodendroglioma and mixed oligoastrocytoma. ${ }^{[1]}$ However, currently there is a paradigm shift in the concept of classification of glial tumors. According to the current revised $4^{\text {th }}$ edition of world health organization (WHO) classification of tumors of central nervous system, classification of gliomas takes into account both morphology and molecular information. ${ }^{[2]}$

Currently gliomas are classified into two basic categories- pilocytic astrocytoma (WHO grade I), and diffuse glioma, which include astrocytomas and oligodendrogliomas (WHO grade II and III) and glioblastoma (GB) (WHO grade IV). In addition, diffuse midline glioma has been included as a distinct molecularly defined entity. These classes have different clinical, morphological and molecular characteristics. We shall discuss the morphological and molecular characteristics of these group of glial tumors along with their significance in this review.

I. Pilocytic astrocytoma (PA): PA is the most common glial tumor seen in children and young patients. It has a predilection for posterior fossa, although other sites such as hypothalamus, cortex are also commonly involved. PA is low grade glial tumor, belongs to grade I.

I A. Histology: PA on histology shows biphasic pattern. There are spindle cells/ bipolar cells (piloid cells) arranged in short fascicles in a fascicular background. These cells have bland nuclear chromatin and elongated cell processes. These are admixed with multipolar cells with a microcystic changes. Mitosis is rare. Areas of infarction, peripheral microvascular proliferation and hyalinised blood vessels are commonly seen. ${ }^{[3]}$ Rosenthal fibres and eosinophilic granular bodies (EGB) are common findings. Rarely, pilocytic astrocytoma can show features of anaplasia. ${ }^{[4]}$
I B. Molecular pathology: Molecular analysis of PA shows alteration of $B R A F$ gene. The most common genetic alteration in $\mathrm{PA}$ is KIAA-BRAF gene fusion. Less commonly (5-10\%) BRAF V600E mutation has been described in PA. ${ }^{[5]}$ There is no known prognostic significance of genetic alteration in PA. Complete surgical removal is the treatment of choice and is usually curative.

II. Diffuse glioma: The group diffuse glioma constitutes grade II and III astrocytoma and oligodendroglioma (ODG), and glioblastoma. Astrocytoma and ODG (grade II and III) share common molecular alteration, such as frequent isocitrate dehydrogenase (IDH) mutation. Thus, they are grouped under same umbrella.

II A. Histology:

II A. 1. Astrocytoma: Astrocytoma includes diffuse astrocytoma (grade II) and anaplastic astrocytoma (grade III), which are defined histologically.

\section{Diffuse astrocytoma (DA):}

DA is characterized by diffuse pattern of growth. These tumors usually have an infiltrative margin with poor demarcation from the adjacent brain parenchyma. The neoplastic astrocytes have elongated nuclei with minimal nuclear atypia. The cells have long cellular processes, which are better appreciated on crush smear. Mitosis is usually sparse. Presence of a few mitoses in a resection specimen of glioma doesn't increase the grade. However, presence of one or more mitosis in a stereotactic biopsy should raise the suspicion of a higher grade. Sometimes, it may be difficult to determine the mitotic activity accurately by light microscopy. MIB labeling index provides valuable information about the proliferative activity of the tumor. Areas with higher labeling index (hot spot) should be re-evaluated for mitotic activity. By definition, there is no necrosis or microvascular proliferation in DA. The stroma is fibrillar. There may be various stromal changes including microcystic changes, myxoid changes, calcification etc. ${ }^{[2]}$ 


\section{International Journal of Innovative Research in Medical Science (IJIRMS) Volume 03 Issue 11 Nov 2018, ISSN: 2455-8737, Imp. Factor - 4.102 Available online at - $\underline{w w w . i j i r m s . i n}$}

There may be variable number of gemistocytes in diffuse glioma. If there is presence of $>20 \%$ gemistocytes, it is defined as gemistocytic astrocytoma. ${ }^{[2]}$ The gemistocytes are round to polygonal cells with eccentrically placed nuclei and abundant, glassy eosinophilic cytoplasm. There is mild to moderate nuclear atypia. Mitosis is usually low. It can show presence of perivascular lymphocytic cuffing. Initially it was claimed that gemistocytic astrocytoma is associated with poor prognosis, however, there is no convincing proof of this. ${ }^{[6]}$ If, a gemistocytic astrocytoma exhibits frequent mitosis, it is considered anaplastic astrocytoma.

\section{Anaplastic astrocytoma:}

Anaplastic astrocytoma (AA) is also characterized by diffuse pattern of growth. The tumor is usually more cellular than diffuse astrocytoma. There may be variable number of gemistocytes. The cells show nuclear pleomorphism, hyperchromatia and coarse chromatin. Mitotic activity is usually brisk. ${ }^{[7]}$ However, in case of stereotactic biopsy, even a single mitotic figure is also considered significant. There is no necrosis or microvascular proliferation (MVP). ${ }^{[8]}$

II A. 2. Oligodendroglioma (ODG): Morphologically this group of tumors shows oligodendroglial differentiation, and constitutes of grade II ODG and anaplastic (grade III) ODG.

ODG can show diffuse pattern of growth, but they can show relatively circumscribed margins. ODGs may be multifocal and cortical based. There are thin, delicately branched blood vessels coursing through the tumor (chicken wire or crow feet blood vessels). ${ }^{[8]}$ The tumor cells in grade II ODG are uniform, with centrally placed round monomorphic nuclei, surrounded by perinuclear halo, giving 'fried-egg' appearance. The tumor cells infiltrate the adjacent cortex, and are arranged around pre-formed structures like neurons and blood vessels. Neurons in the adjacent cortex are surrounded by the tumor cells (perineuronal satellitosis). Scattered small gemistocytes (mini gemistocytes) are seen within the tumor, however, in rare cases they are present in abundance. There may be few mitoses (less than 6/ $10 \mathrm{HPF}$ ). Necrosis and MVP are absent in grade II ODG. Secondary changes include calcification and microcystic changes. ${ }^{[9]}$

Grade III (anaplastic) ODG shows increased cellularity and nuclear atypia. The tumor cells show coarse nuclear chromatin. Mitotic activity is brisk ( $\geq 6 / 10 \mathrm{HPF}$ ). There may be presence of necrosis and MVP. Finding necrosis and MVP in a tumor showing classical ODG morphology and ODG like molecular findings does not warrant a diagnosis of glioblastoma. ${ }^{[9]}$

II A. 3. Glioblastoma: Glioblastoma (GB) is the commonest and most aggressive glial neoplasm. It is considered WHO grade IV.

GB is highly cellular tumor, composed of neoplastic astrocytes. The histopathology of GB is highly variable. The conventional GB is composed of poorly differentiated, round, polygonal to elongated cells, with scanty to moderate amount of cytoplasm. The cells show moderate to severe nuclear pleomorphism and coarse chromatin. There may be variable number of giant cells and gemistocytes. Mitosis is usually brisk. GB is characterized by presence of necrosis and or microvascular proliferation (MVP). The necrosis can be small, palisaded by tumor cells (pseudopalisading necrosis) or it may be large, geographic area of necrosis. GB cells can show spindling focally, more so when it infiltrates the meninges. GB can show presence of variable cellular patterns.

Small cell glioblastoma: It is composed of relatively uniform, small size cells with little cytoplasm. These cells show monomorphic dark nuclei, but brisk mitosis. They can have delicate, branching vessel, thus resembling oligodendroglioma. However, in contrast to ODG, small cell GB shows loss of chromosome $10 \mathrm{q}$ and doesn't show $1 \mathrm{p} / 19 \mathrm{q}$ co-deletion. ${ }^{[10]}$

Glioblastoma with a primitive neuronal component: This pattern shows conventional GB morphology, with areas of undifferentiated cellular component. These foci of undifferentiated cells are highly cellular and are sharply demarcated from the adjacent conventional GB. These are composed of undifferentiated cells with high $\mathrm{N}: \mathrm{C}$ ratio, hyperchromatic nuclei and brisk mitosis. These cells can show wrapping and presence of Homer Wight rosettes, resembling other embryonal neoplasms. The primitive component shows high frequency of $\mathrm{MYCN}$ or MYC gene amplification. This variant is associated with aggressive behavior and shows frequent cerebrospinal fluid dissemination. ${ }^{[11]}$

Glioblastoma with oligodendroglioma component: Occasional glioblastoma contains areas which may resemble classical ODG. These ODG like component may be focal or extensive. When this is the predominant component, it should be differentiated from anaplastic oligodendroglioma by use of $1 \mathrm{p} / 19 \mathrm{q}$ fluorescence in situ hybridization (FISH), which by definition, should be negative in GB with oligodendroglioma component. ${ }^{[12]}$

Granular cell glioblastoma: These cases contain variable number of cells with abundant granular cytoplasm. These cells may be scattered or abundant. The cytoplasm shows strong periodic acid Schiff (PAS) positivity. These cells may resemble macrophages. On ultrastructural examination, these cells are rich in lysozymes. These granular cells are mostly negative for glial fibrillary acidic protein (GFAP), but some may show focal GFAP expression. The granular cells often express CD68. ${ }^{[13]}$

Lipid rich glioblastoma: Some cases of GB show presence of heavily lipidized or foam cells. These lesions should be differentiated from pleomorphic xanthoastrocytoma, which can resemble it histologically. ${ }^{[14]}$

Metaplastic changes: GBs can show various metaplastic changes. The metaplasia can be both epithelial as well as mesenchymal. The changes are more common in gliosarcoma than conventional GBs. The epithelial metaplasia can be present in the form of squamous epithelium. Some cases show epithelial cells arranged in the form of glands and ribbon, with or without abundant mucin production. These cases are also known as adenoid GB. These cases usually show presence of conventional GB component, at least focally. The epithelial cells express cytokeratin and negative for GFAP. These cases need to be distinguished from metastatic carcinoma. Among the mesenchymal elements, bone and cartilage have been described as metaplastic component.

Gemistocyte rich glioblastoma: Some cases of GB can show numerous gemistocytes. 


\section{International Journal of Innovative Research in Medical Science (IJIRMS) Volume 03 Issue 11 Nov 2018, ISSN: 2455-8737, Imp. Factor - 4.102 Available online at - $\underline{w w w . i j i r m s . i n}$}

Morphological variants of glioblastoma: Apart from conventional glioblastoma with various cellular components, three distinct morphological variants of GB are recognized.

Epithelioid glioblastoma (eGB): This is a rare variant of GB and more commonly occurs in younger individuals. eGB is composed of discohesive sheets of large, round to polygonal epithelioid cells with abundant eosinophilic cytoplasm. These cells show pleomorphic nuclei. Some cells may show intracytoplasmic inclusion, resembling rhabdoid cells. However, these cells show retained nuclear INI1 expression, thus helps to differentiate from atypical teratoid/ rhabdoid tumor (AT/RT). These tumor cells can show patchy epithelial membrane antigen (EMA) expression, while GFAP expression is variable. BRAF V600E mutation is identified in around $50 \%$ of eGB, which can be detected using immunohistochemistry. ${ }^{[15,16]}$

Giant cell GB: This variant is rich in multinucleated giant cells. These giant cells are bizarre, contain few to $>20$ nuclei. Some cells show presence of intracytoplasmic inclusion. The tumor cells usually show diffuse and strong p53 expression, while GFAP expression is variable. ${ }^{[17]}$

Gliosarcoma: Gliosarcoma is a biphasic tumor composed of neoplastic glial component and sarcomatous component. The glial component usually shows features of conventional glioblastoma. The glial component is intimately associated with sarcomatous component, composed of spindle cells. The sarcomatous component can be highly cellular, or less cellular with abundant collagen production. The sarcomatous component can show presence of other heterologous components like bone and cartilage. The glial component shows variable GFAP expression, while the sarcomatous component is usually negative for GFAP and shows vimentin expression. The sarcomatous component is rich in reticulin. $^{[18]}$

\section{B. Molecular pathology of diffuse glioma:}

The understanding about the pathogenesis and biology of gliomas has undergone revolutionary changes in the recent years. The incorporation of molecular information has helped categorization of glial tumors into different prognostic groups, which assists the individualization and optimization of the treatment. Isocitrate dehydrogenase (IDH) is one of the most well recognized and widely described molecular markers in diffuse gliomas, both with astrocytic and oligodendroglial differentiation. Mutations in IDH 1 and 2 genes, originally discovered in 2009, occur in the vast majority of diffuse gliomas (grade II and III), frequency ranging from $54 \%$ to $90 \% .^{[19,20,21]} \mathrm{IDH} 1$ mutation is most common, and it is seen in $\sim 80 \%$ of grade II-III gliomas and secondary GB. Mutations in $I D H 2$ have also been identified in diffuse gliomas, although they are much less common $(<3 \%)$ and are mutually exclusive with IDH1 mutations. ${ }^{[22,23]}$ All mutations identified to date have been a single amino acid missense mutation in IDHI at arginine 132 (R132) or the analogous residue in $I D H 2$ (R172). Although $I D H$ mutation is a hallmark of diffuse glioma, it is almost never seen in pilocytic astrocytoma and primary glioblastoma $(<10 \%) .{ }^{[20]}$ Based on $I D H$ mutation status, astrocytoma and oligodendroglioma are classified as IDH mutant and IDH wildtype, according to the current WHO classification of brain tumors. ${ }^{[2]}$ Among the different IDH1 mutations, the commonest is $\mathrm{R} 132 \mathrm{H}$ mutation at codon position 132 , seen in $>90 \%$ cases. Other mutations in this position are less frequent. ${ }^{[20,23]}$ Although sequencing is the gold standard to detect IDH1 mutation, commercially available antibody against IDH1 R132H mutant protein shows high sensitivity, specificity, and correlates very well with sequencing results. ${ }^{[24,25,26]} I D H 2$ mutation is much less frequent than $I D H I$ mutation, and is more commonly seen in oligodendroglial tumors compared to astrocytomas. $^{[27,28,29,30]}$

Exactly how IDH mutation contributes to oncogenic transformation remains controversial. Mutated IDH1 protein acquires the ability to convert $\alpha$-ketogluterate $(\alpha-K G)$ to $R(-)-2-$ hydroxyglutarate (2-HG). 2-HG levels are elevated in gliomas containing IDHI mutation. These findings led to the hypothesis that mutant $I D H$ is an oncogene and 2-HG is an 'oncometabolite'. ${ }^{[31]}$ The increased levels of 2-hydroxyglutarate in IDH mutated tumors have inspired several groups to develop noninvasive imaging methods for detection of $I D H$-mutated gliomas. 2-HG has a distinctive magnetic resonance spectrum that is detectable by in vivo magnetic resonance spectroscopy (MRS). MRS may therefore be useful for distinguishing gliosis from low grade gliomas.

ATRX mutation is a hallmark of astrocytic differentiation, which can be detected by loss of nuclear ATRX expression on immunohistochemistry. Thus, presence of ATRX mutation as detected by loss of nuclear ATRX expression is very sensitive for determining astrocytic phenotype in a diffusely infiltrating grade II and III glioma. ATRX mutation is found frequently in DA (60$70 \%)$ and AA (70-80\%) and is rare in primary GB $(4-6 \%) .{ }^{[32,33]}$ On the other hand, co-deletion of chromosome $1 \mathrm{p}$ and $19 \mathrm{q}$ is diagnostic hallmark of oligodendroglial differentiation. ${ }^{[34]}$ Fluorescent in situ hybridization (FISH) is the preferred and most widely used tool for detecting $1 \mathrm{p} / 19 \mathrm{q}$ co-deletion. ATRX mutation and $1 \mathrm{p} / 19 \mathrm{q}$ co-deletion are almost mutually exclusive. ${ }^{[32]}$ ATRX status combined with $1 \mathrm{p} / 19 \mathrm{q}$ and $I D H 1 / I D H 2$ mutation status has been used to generate a molecular diagnostic algorithm for diffuse gliomas, which has been shown to be superior to conventional morphological classification. Diffuse glioma with $I D H$ mutation, $1 \mathrm{p} / 19 \mathrm{q}$ co-deletion, and retained ATRX expression has been diagnosed as oligodendroglioma and tumors with $I D H$ mutation, intact $1 \mathrm{p} / 19 \mathrm{q}$ and loss of nuclear ATRX expression has been classified as astrocytomas. ${ }^{[33]}$ This molecular classification of gliomas based on gene signature has got profound therapeutic and prognostic significance, as discussed subsequently. Since ATRX mutation and $1 \mathrm{p} / 19 \mathrm{q}$ co-deletion are almost mutually exclusive, all diffuse gliomas should be initially screened by IHC for IDH1 R132H and ATRX. Cases showing astrocytic morphology and loss of nuclear ATRX can be safely diagnosed as astrocytoma, whereas cases showing classical ODG phenotype, IDH positivity and retained nuclear ATRX expression, can be safely diagnosed as oligodendroglioma. More expensive investigation such as FISH for $1 \mathrm{p} / 19 \mathrm{q}$ can be avoided in those patients. However, cases showing astrocytic morphology but retained ATRX expression and cases showing ODG like morphology, but showing loss of ATRX with or without p53 over-expression should be subjected to $1 \mathrm{p} / 19 \mathrm{q}$ co-deletion status for accurate molecular classification (figure 1). This algorithm also shows high sensitivity and specificity to classify diffuse gliomas into molecular subgroups, according to WHO 2016 classification. ${ }^{[35]}$ BRAF V600E mutation and BRAF gene fusion are extremely rare in diffuse gliomas. ${ }^{[26]}$ 
International Journal of Innovative Research in Medical Science (IJIRMS)

Volume 03 Issue 11 Nov 2018, ISSN: 2455-8737, Imp. Factor - 4.102

Available online at $-\underline{w w w . i j i r m s . i n}$

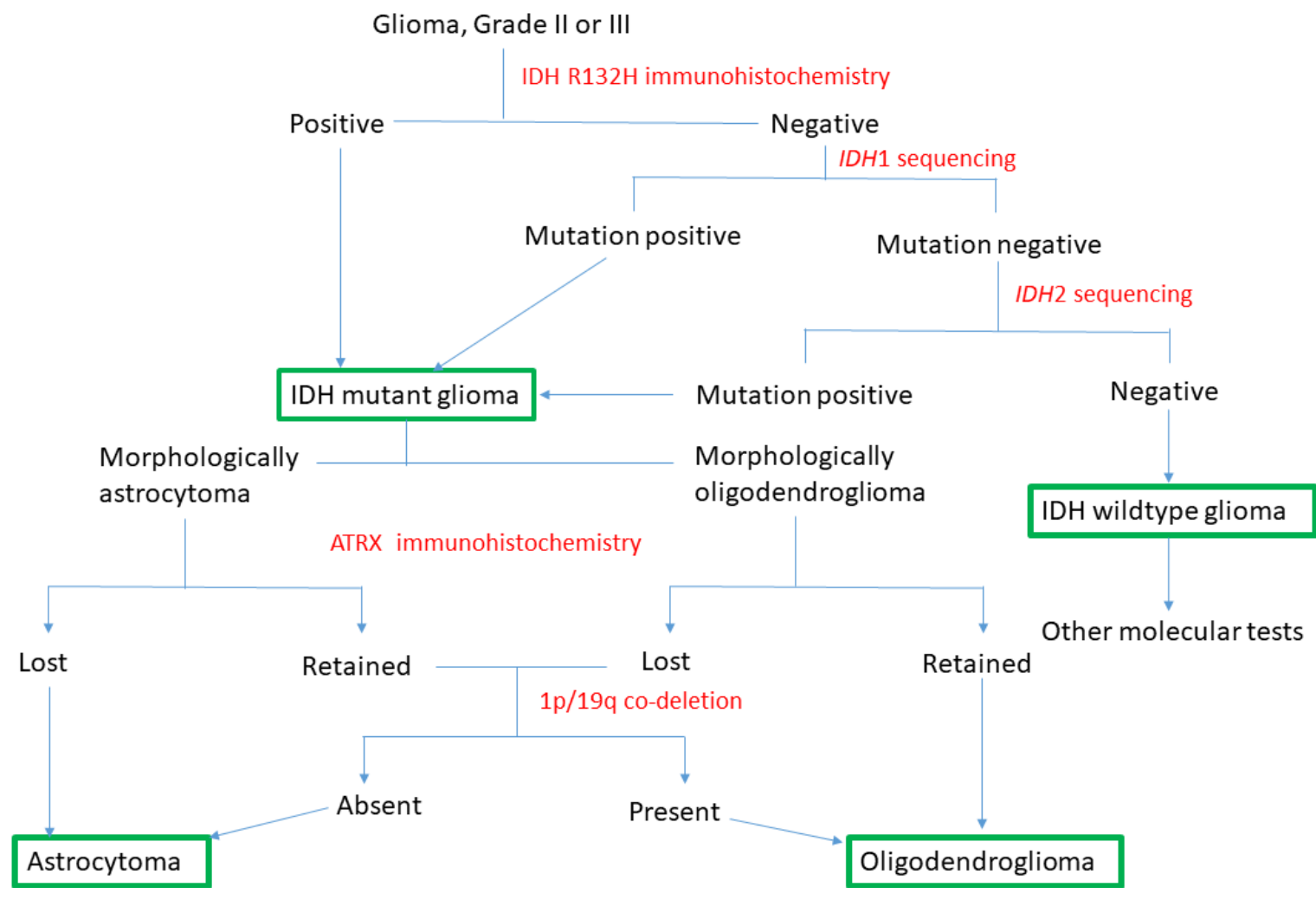

Figure 1: Simplified diagnostic approach to histological grade II/III gliomas

Molecular pathology of glioblastoma: Classically GB was further divided as primary and secondary glioblastomas based on their clinical and molecular features. ${ }^{[36,37]}$ Primary glioblastomas arise de novo and seen in older individuals whereas secondary glioblastomas arise from pre-existing low-grade gliomas and seen in younger patients (table 1). Patients with secondary glioblastomas experience longer survival compared to primary GB. Currently, based on the molecular pathology, GB is classified into two main groups, IDH wildtype and IDH mutated, which roughly corresponds to primary and secondary GB respectively. ${ }^{[36,37]}$ Major molecular alterations classically described in IDH wildtype GB include telomerase reverse transcriptase promoter (TERTp) mutation (seen up to $80 \%$ cases), EGFR amplification, PTEN mutation and $\mathrm{LOH} 10 \mathrm{p}$ and 10q. ${ }^{[38]}$ Receptor tyrosine kinase signaling can be brought about by copy number alteration or by altered receptor signaling. High copy amplicons often occur in regions with known oncogenes (EGFR, MDM2 and CDK4), but may also occur in other regions. Frequent intermediate copy number gains or losses include trisomy of chromosome 7 (42\%) and monosomy of chromosome $10(58 \%)$, which are associated with a poor prognosis. The receptor tyrosine kinase (RTK) signaling pathway is involved in the translation of growth factor signals into increased proliferation and survival. ${ }^{[39,40]}$ The most frequently altered gene in the RTK pathway is EGFR. It is amplified in up to $45 \%$ of glioblastomas and results in an increase in mRNA and protein expression. In addition to amplification, $E G F R$ is often constitutively activated by variants including EGFRVIII in which exons $2-7$ are deleted. ${ }^{[41]}$ Other RTKs may also be affected in GBM and include PDGFRA (amplified in 13\%), ERBB2 (mutated in $8 \%$ ) and $C M E T$ (amplified in $4 \%$ ). ${ }^{[42]}$ In contrast to primary glioblastoma, secondary glioblastomas (IDH mutant GB) harbour many genetic alterations that are commonly seen in diffuse gliomas, such as IDH and ATRX. P53 mutation is more common in IDH mutant GB compared to IDH wildtype GB. In contrast to IDH wildtype GB, TERTp mutation is uncommon and EGFR amplification and PTEN mutations are extremely rare in IDH mutant GB. ${ }^{[2]}$

Table 1: Comparison of primary and secondary glioblastoma

\begin{tabular}{|l|l|l|}
\hline Features & Primary glioblastoma & Secondary glioblastoma \\
\hline Proportion of cases & $>90 \%$ & $<10 \%$ \\
\hline Median age of onset & $7^{\text {th }}$ decade & $5^{\text {th }}$ decade \\
\hline Precursor lesion & None identified & Grade II and III astrocytoma \\
\hline Duration of symptoms & $<6$ months, rapid onset & $12-18$ months, slowly progressive \\
\hline Median overall survival & 1.2 years & 2.3 years \\
\hline EGFR amplification & $30-40 \%$ & Very rare \\
\hline$T E R T$ p mutation & $70-80 \%$ & $20-30 \%$ \\
\hline$P T E N$ mutation & $20-30 \%$ & Very rare \\
\hline$I D H$ mutation & Exceptional & $80-90 \%$ \\
\hline$T P 53$ mutation & $25-30 \%$ & $80-90 \%$ \\
\hline$A T R X$ mutation & Exceptional & $70-75 \%$ \\
\hline
\end{tabular}


II C. Prognostic significance of molecular markers in diffuse gliomas:

It is well known that oligodendroglioma (as defined by $1 \mathrm{p} / 19 \mathrm{q}$ codeletion) carries better prognosis than astrocytomas. IDH mutation is associated with favorable outcome in high grade gliomas. ${ }^{[43,44]}$ Weller et al. examined IDHI mutation in $301 \mathrm{~GB}$ patients, and demonstrated that $I D H 1$ mutation in GB is associated with better progression free and overall survival. ${ }^{[44]}$ While the prognostic role of $I D H$ mutation in high grade glioma is unequivocally established, there is confusion regarding its prognostic value in the low grade glial tumors. Sanson et al. observed that $I D H 1$ mutated DA show longer overall survival than IDHI wild cases (150.9 vs. 60.1 months). ${ }^{[45]}$ Other studies have also demonstrated that $I D H$ mutation in grade II gliomas predicts better overall and progression free survival. ${ }^{[21,46]}$ However, some studies demonstrated conflicting result and found no association of $I D H$ mutation with progression free or overall survival in low grade gliomas. ${ }^{[27,47]}$ Ruess et al. found that $I D H$ mutation in astrocytomas carries more prognostic significance compared to their histological grade. IDH mutant DA and AA cases in their study had little difference in survival. ${ }^{[48]}$ This may be a fallacy of the current WHO grading system of astrocytomas. Currently IDH inhibitors have been tried in the treatment of IDH mutant gliomas. In vitro and in vivo studies involving animal models have shown promising result. IDH inhibitors have been shown to inhibit the growth of IDH mutant glioma cells in culture, although all mutant cell lines don't show similar results. ${ }^{[49]}$ Thus, IDH inhibitors may emerge as an effective treatment option for gliomas in the future.

IDH wild DA and AA are provisional entities in current WHO classification. They are considered as a heterogeneous group of tumors and majority of these cases show molecular features of glioblastoma. However, prognosis in this group is determined by other molecular alterations like TERTp mutation, EGFR amplification, MYB amplification, and H3F3A mutation. Further studies are required to better characterize and understand the nature of IDH wild type diffuse gliomas. ${ }^{[50,51]}$

Glioblastoma shows an aggressive disease course and is associated with poor outcome. It is well established that IDH mutated glioblastoma has a much better prognosis compared to IDH wildtype GB. Different studies have found increased overall survival of IDH mutant GB compared to IDH wildtype GB (2.3 years vs 1.2 years) ${ }^{[52]}$ Glioblastoma is usually treated with various combination of surgery, radiotherapy and chemotherapy. Temozolomide has been proven effective in improving the median overall survival in glioblastoma by few months, and is most effective in those in which the MGMT promoter is hypermethylated. ${ }^{[53]}$ TERT promoter mutation is detected in 7595\% of the IDH wildtype glioblastoma denotes a poor prognosis. ${ }^{[5,55,56]}$ TERTp mutation is fairly common in IDH mutant oligodendrogliomas $(>90 \%)$, while it is rare in astrocytomas $(<10 \%) \cdot{ }^{[54]}$ Gliomas harbouring only TERT promoter mutation are predominantly grade IV tumors. Based on IDH mutation, 1p/19q co-deletion and TERT promoter mutation status, diffuse gliomas have been classified into different prognostic groups. These groups include (a) triple positive (IDH mutant, 1p/19q co-deleted and TERTp mutated), (b) IDH and TERTp mutated, (c) Only IDH mutated, (d) only TERTp mutated, and (e) triple negative. For grade II and III gliomas, group showing both TERTp and IDH mutation shows best survival, followed by only
IDH mutant group. Only TERTp mutated group had worst survival in grade II and III gliomas. This is contrast to glioblastomas, where IDH mutant only group had best survival and group showing TERT only mutated had the worst outcome, similar to the group showing both IDH and TERTp mutation. ${ }^{[57]}$ Similar findings have been described in other studies as well. ${ }^{[58]}$ These findings indicate that there is a complex interplay of different molecular markers determining the prognosis in glioma with different histological grades. In grade II and III gliomas, IDH mutation with or without TERT mutation indicates a good prognosis, whereas in glioblastoma, combined IDH and TERT mutation is associated with a bleak prognosis, while only IDH mutant group shows the best coutcome. Thus, IDH mutation is not always associated with a favorable outcome. Arita et al. in a recent study described TERTp mutation is associated with a better overall survival in MGMT methylated GB, compared to TERTp wildtype cases. ${ }^{[59]}$ Thus there is a complex interaction among the different prognostic genetic markers, and no single molecular marker in isolation is able to determine the prognosis precisely.

\section{Diffuse midline glioma:}

Diffuse midline glioma is a diffusely infiltrating glial neoplasm, arising in the midline. It is usually seen in children and most commonly shows astrocytic differentiation. The midline structures such as thalamus, brain stem and spinal cord are the most common sites of occurrence. Few cases of pineal gland origin have also been described. ${ }^{[60]}$

Morphology: It usually shows diffuse pattern of growth with astrocytic morphology. The cells are oval to elongated, although oligodendroglial morphology can also be seen in minority of cases. A wide morphological spectrum can be obtained. These tumors usually show high grade features, such as frequent mitosis, necrosis and endovascular proliferation. However, high grade histological features are not recognizable in up to $30 \%$ cases. ${ }^{[61]}$ Morphologically, the low grade tumors may resemble diffuse astrocytoma, ganglioglioma or pilocytic astrocytoma. ${ }^{[62]}$

Molecular genetics: This group of tumors is characterized by recurrent mutation of histone coding genes $H 3 F 3 A, H I S T 1 H 3 B$ and HISTIH3C at position K27. The frequency of these mutations in diffuse midline glioma ranges from $60-80 \%$. The most common mutation is $\mathrm{K} 27 \mathrm{M}$ mutation in the H3F3A gene (H3K27M). Presence of this mutation can be detected by immunohistochemistry using mutant specific antibody. H3K27M mutated diffuse midline glioma shows uniformly poor prognosis, irrespective of their location. ${ }^{[63]}$ Thus H3K27M mutated diffuse midline gliomas are regarded grade IV tumor, irrespective of their histological pattern.

Role of histology in the current era of molecular classification of gliomas: In the current era, molecular pathology is being increasingly used for the diagnosis and classification of gliomas. There is raising concern whether histological examination of the tumor will remain relevant in the diagnosis and management of the glioma patients. As molecular testing is applied more frequently to the diagnosis of brain tumors, inconsistent or conflicting molecular information will create diagnostic challenge. It should be remembered that although the molecular signature can accurately classify the glial tumors in most of the cases, there may be discrepant molecular findings and some tumors remain unclassifiable. For example, small percentage of tumors can 


\section{International Journal of Innovative Research in Medical Science (IJIRMS) \\ Volume 03 Issue 11 Nov 2018, ISSN: 2455-8737, Imp. Factor - 4.102 \\ Available online at $-\underline{w w w . i j i r m s . i n}$}

harbour both $A T R X$ mutation and 1p/19q co-deletion. These cases are ultimately managed based on histology. ${ }^{[64]}$ And till date, the grade of glioma is still based on its histological features (except for diffuse midline glioma with $H 3 K 27 M$ mutation, which assign it grade IV irrespective of morphology). Most of the grade I tumors are diagnosed based on histology and don't require molecular investigations. Thus histology is still remains important part of management of glioma patients.

\section{Conclusion:}

Gliomas are heterogeneous disease, both histologically as well as at their genetic level. Although gliomas are diagnosed mainly based on their histology, molecular investigation has become an essential part of their work up and management. Molecular features not only help in accurate diagnosis of glioma, they also provide valuable prognostic and predictive information.

\section{Conflict of interest: Nil}

\section{Source of funding: None}

\section{References:}

[1] Louis DN, Ohgaki H, Wiestler OD, Cavenee WK, editors. In: WHO Classification of Tumours of the Central Nervous System. Fourth edition. International Agency for Research on Cancer (IARC): Lyon; 2007.

[2] Louis DN, Ohgaki H, Wiestler OD, Cavenee WK, editors. In: WHO Classification of Tumours of the Central Nervous System. Revised fourth edition. International Agency for Research on Cancer (IARC): Lyon; 2016.

[3] Matyja E, Grajkowska W, Stepien K, Naganska E. Heterogeneity of histopathological presentation of pilocytic astrocytoma - diagnostic pitfalls. A review. Folia Neuropathol. 2016;54(3):197-211.

[4] Yong EX, McKelvie P, Murphy M, Wang YY. Anaplastic pilocytic astrocytoma. J Clin Neurosci. 2014;21(11):1993-6.

[5] Collins VP, Jones DT, Giannini C. Pilocytic astrocytoma: pathology, molecular mechanisms and markers. Acta Neuropathol. 2015;129(6):775-88.

[6] Tihan T, Vohra P, Berger MS, Keles GE. Definition and diagnostic implications of gemistocytic astrocytomas: a pathological perspective. J Neurooncol. 2006;76(2):17583.

[7] Grimm SA, Chamberlain MC. Anaplastic astrocytoma. CNS Oncol. 2016;5(3):145-57.

[8] Perry A, Wesseling P. Histologic classification of gliomas. Handb Clin Neurol. 2016;134:71-95.

[9] Wesseling $P$, van den Bent M, Perry A. Oligodendroglioma: pathology, molecular mechanisms and markers. Acta Neuropathol. 2015;129(6):809-27.

[10] Takeuchi H, Kitai R, Hosoda T, Yamada S, Hashimoto N, Kikuta K, et al. Clinicopathologic features of small cell glioblastomas. J Neurooncol. 2016;127(2):337-44.

[11] Konar SK, Bir SC, Maiti TK, Patra DP, DiPoto Brahmbhatt AC, Jacobsohn JA, et al. Early dural metastasis from a case of glioblastoma with primitive neuroectodermal differentiation: A case report and literature review. J Clin Neurosci. 2017;35:78-81.

[12] Wang Y, Li S, Chen L, You G, Bao Z, Yan W, et al. Glioblastoma with an oligodendroglioma component: distinct clinical behavior, genetic alterations, and outcome. Neuro Oncol. 2012;14(4):518-25.

[13] Joo M, Park SH, Chang SH, Kim H, Choi CY, Lee CH, et al. Cytogenetic and molecular genetic study on granular cell glioblastoma: a case report. Hum Pathol. 2013;44(2):282-8.

[14] Singh AD, Iftinca M, Easaw JC. Lipidized glioblastoma: pathological and molecular characteristics. Neuropathology. 2013;33(1):87-92.

[15] Broniscer A, Tatevossian RG, Sabin ND, Klimo P, Jr., Dalton J, Lee R, et al. Clinical, radiological, histological and molecular characteristics of paediatric epithelioid glioblastoma. Neuropathol Appl Neurobiol. 2014;40(3):327-36.

[16] Kleinschmidt-DeMasters BK, Aisner DL, Birks DK, Foreman NK. Epithelioid GBMs show a high percentage of BRAF V600E mutation. Am J Surg Pathol. 2013;37(5):685-98.

[17] Kozak KR, Moody JS. Giant cell glioblastoma: a glioblastoma subtype with distinct epidemiology and superior prognosis. Neuro Oncol. 2009;11(6):833-41.

[18] Han SJ, Yang I, Tihan T, Prados MD, Parsa AT. Primary gliosarcoma: key clinical and pathologic distinctions from glioblastoma with implications as a unique oncologic entity. J Neurooncol. 2010;96(3):313-20.

[19] Ichimura K, Pearson DM, Kocialkowski S, Backlund LM, Chan R, Jones DT, et al. IDH1 mutations are present in the majority of common adult gliomas but rare in primary glioblastomas. Neuro Oncol. 2009;11(4):3417.

[20] Watanabe T, Nobusawa S, Kleihues P, Ohgaki H. IDH1 mutations are early events in the development of astrocytomas and oligodendrogliomas. Am J Pathol. 2009;174(4):1149-53.

[21] Zou P, Xu H, Chen P, Yan Q, Zhao L, Zhao P, et al. IDH1/IDH2 mutations define the prognosis and molecular profiles of patients with gliomas: a metaanalysis. PLoS One. 2013;8(7):e68782.

[22] Cohen AL, Holmen SL, Colman H. IDH1 and IDH2 mutations in gliomas. Curr Neurol Neurosci Rep. 2013;13(5):345.

[23] Yan H, Parsons DW, Jin G, McLendon R, Rasheed BA, Yuan W, et al. IDH1 and IDH2 mutations in gliomas. N Engl J Med. 2009;360(8):765-73.

[24] Capper D, Zentgraf H, Balss J, Hartmann C, von Deimling A. Monoclonal antibody specific for IDH1 R132H mutation. Acta Neuropathol. 2009;118(5):599601.

[25] Capper D, Weissert S, Balss J, Habel A, Meyer J, Jager $\mathrm{D}$, et al. Characterization of R132H mutation-specific IDH1 antibody binding in brain tumors. Brain Pathol. 2010;20(1):245-54.

[26] Chatterjee D, Radotra BD, Kumar N, Vasishta RK, Gupta SK. IDH1, ATRX, and BRAFV600E mutation in astrocytic tumors and their significance in patient outcome in north Indian population. Surg Neurol Int. 2018;9:29. 


\section{International Journal of Innovative Research in Medical Science (IJIRMS) Volume 03 Issue 11 Nov 2018, ISSN: 2455-8737, Imp. Factor - 4.102 \\ Available online at $-\underline{w w w . i j i r m s . i n}$}

[27] Ahmadi R, Stockhammer F, Becker N, Hohlen K, Misch $\mathrm{M}$, Christians A, et al. No prognostic value of IDH1 mutations in a series of $100 \mathrm{WHO}$ grade II astrocytomas. J Neurooncol. 2012;109(1):15-22.

[28] Cahill DP, Sloan AE, Nahed BV, Aldape KD, Louis DN, Ryken TC, et al. The role of neuropathology in the management of patients with diffuse low grade glioma: A systematic review and evidence-based clinical practice guideline. J Neurooncol. 2015;125(3):531-49.

[29] Hartmann C, Meyer J, Balss J, Capper D, Mueller W, Christians A, et al. Type and frequency of IDH1 and IDH2 mutations are related to astrocytic and oligodendroglial differentiation and age: a study of 1,010 diffuse gliomas. Acta Neuropathol. 2009;118(4):469-74.

[30] Zhang CB, Bao ZS, Wang HJ, Yan W, Liu YW, Li MY, et al. Correlation of IDH1/2 mutation with clinicopathologic factors and prognosis in anaplastic gliomas: a report of 203 patients from China. J Cancer Res Clin Oncol. 2014;140(1):45-51.

[31] Mustafa DA, Swagemakers SM, Buise L, van der Spek PJ, Kros JM. Metabolic alterations due to IDH1 mutation in glioma: opening for therapeutic opportunities? Acta Neuropathol Commun. 2014;2:6.

[32] Jiao Y, Killela PJ, Reitman ZJ, Rasheed AB, Heaphy CM, de Wilde RF, et al. Frequent ATRX, CIC, FUBP1 and IDH1 mutations refine the classification of malignant gliomas. Oncotarget. 2012;3(7):709-22.

[33] Wiestler B, Capper D, Holland-Letz T, Korshunov A, von Deimling A, Pfister SM, et al. ATRX loss refines the classification of anaplastic gliomas and identifies a subgroup of IDH mutant astrocytic tumors with better prognosis. Acta Neuropathol. 2013;126(3):443-51.

[34] Cahill DP, Louis DN, Cairncross JG. Molecular background of oligodendroglioma: 1p/19q, IDH, TERT, CIC and FUBP1. CNS Oncol. 2015;4(5):287-94.

[35] Reuss DE, Sahm F, Schrimpf D, Wiestler B, Capper D, Koelsche C, et al. ATRX and IDH1-R132H immunohistochemistry with subsequent copy number analysis and IDH sequencing as a basis for an "integrated" diagnostic approach for adult astrocytoma, oligodendroglioma and glioblastoma. Acta Neuropathol. 2015;129(1):133-46.

[36] Louis DN, Perry A, Reifenberger G, von Deimling A, Figarella-Branger D, Cavenee WK, et al. The 2016 World Health Organization Classification of Tumors of the Central Nervous System: a summary. Acta Neuropathol. 2016;131(6):803-20.

[37] Ohgaki H, Kleihues P. The definition of primary and secondary glioblastoma. Clin Cancer Res. 2013;19(4):764-72.

[38] Aldape K, Zadeh G, Mansouri S, Reifenberger G, von Deimling A. Glioblastoma: pathology, molecular mechanisms and markers. Acta Neuropathol. 2015;129(6):829-48.

[39] Bralten LB, French PJ. Genetic alterations in glioma. Cancers (Basel). 2011;3(1):1129-40.

[40] Houillier C, Lejeune J, Benouaich-Amiel A, LaigleDonadey F, Criniere E, Mokhtari K, et al. Prognostic impact of molecular markers in a series of 220 primary glioblastomas. Cancer. 2006;106(10):2218-23.
[41] Frederick L, Eley G, Wang XY, James CD. Analysis of genomic rearrangements associated with EGRFvIII expression suggests involvement of Alu repeat elements. Neuro Oncol. 2000;2(3):159-63.

[42] Beroukhim R, Getz G, Nghiemphu L, Barretina J, Hsueh $\mathrm{T}$, Linhart $\mathrm{D}$, et al. Assessing the significance of chromosomal aberrations in cancer: methodology and application to glioma. Proc Natl Acad Sci U S A. 2007;104(50):20007-12.

[43] Takano S, Tian W, Matsuda M, Yamamoto T, Ishikawa E, Kaneko MK, et al. Detection of IDH1 mutation in human gliomas: comparison of immunohistochemistry and sequencing. Brain Tumor Pathol. 2011;28(2):115-23.

[44] Weller M, Felsberg J, Hartmann C, Berger H, Steinbach JP, Schramm J, et al. Molecular predictors of progression-free and overall survival in patients with newly diagnosed glioblastoma: a prospective translational study of the German Glioma Network. J Clin Oncol. 2009;27(34):5743-50.

[45] Sanson M, Marie Y, Paris S, Idbaih A, Laffaire J, Ducray $\mathrm{F}$, et al. Isocitrate dehydrogenase 1 codon 132 mutation is an important prognostic biomarker in gliomas. J Clin Oncol. 2009;27(25):4150-4.

[46] Houillier C, Wang X, Kaloshi G, Mokhtari K, Guillevin $\mathrm{R}$, Laffaire $\mathrm{J}$, et al. IDH1 or IDH2 mutations predict longer survival and response to temozolomide in lowgrade gliomas. Neurology. 2010;75(17):1560-6.

[47] Thon N, Eigenbrod S, Kreth S, Lutz J, Tonn JC, Kretzschmar $\mathrm{H}$, et al. IDH1 mutations in grade II astrocytomas are associated with unfavorable progression-free survival and prolonged postrecurrence survival. Cancer. 2012;118(2):452-60.

[48] Reuss DE, Mamatjan Y, Schrimpf D, Capper D, Hovestadt V, Kratz A, et al. IDH mutant diffuse and anaplastic astrocytomas have similar age at presentation and little difference in survival: a grading problem for WHO. Acta Neuropathol. 2015;129(6):867-73.

[49] Kopinja J, Sevilla RS, Levitan D, Dai D, Vanko A, Spooner E, et al. A Brain Penetrant Mutant IDH1 Inhibitor Provides In Vivo Survival Benefit. Sci Rep. 2017;7(1):13853.

[50] Aibaidula A, Chan AK, Shi Z, Li Y, Zhang R, Yang R, et al. Adult IDH wild-type lower-grade gliomas should be further stratified. Neuro Oncol. 2017;19(10):1327-37.

[51] Reuss DE, Kratz A, Sahm F, Capper D, Schrimpf D, Koelsche C, et al. Adult IDH wild type astrocytomas biologically and clinically resolve into other tumor entities. Acta Neuropathol. 2015;130(3):407-17.

[52] Ohno M, Narita Y, Miyakita Y, Matsushita Y, Arita H, Yonezawa $\mathrm{M}$, et al. Glioblastomas with IDH1/2 mutations have a short clinical history and have a favorable clinical outcome. Jpn J Clin Oncol. 2016;46(1):31-9.

[53] Binabaj MM, Bahrami A, ShahidSales S, Joodi M, Joudi Mashhad M, Hassanian SM, et al. The prognostic value of MGMT promoter methylation in glioblastoma: A meta-analysis of clinical trials. J Cell Physiol. 2018;233(1):378-86.

[54] Lee Y, Koh J, Kim SI, Won JK, Park CK, Choi SH, et al. The frequency and prognostic effect of TERT promoter 
mutation in diffuse gliomas. Acta Neuropathol Commun. 2017;5(1):62.

[55] Jeong DE, Woo SR, Nam H, Nam DH, Lee JH, Joo KM. Preclinical and clinical implications of TERT promoter mutation in glioblastoma multiforme. Oncol Lett. 2017;14(6):8213-9.

[56] Mosrati MA, Malmstrom A, Lysiak M, Krysztofiak A, Hallbeck M, Milos $\mathrm{P}$, et al. TERT promoter mutations and polymorphisms as prognostic factors in primary glioblastoma. Oncotarget. 2015;6(18):16663-73.

[57] Eckel-Passow JE, Lachance DH, Molinaro AM, Walsh KM, Decker PA, Sicotte H, et al. Glioma Groups Based on $1 \mathrm{p} / 19 \mathrm{q}$, IDH, and TERT Promoter Mutations in Tumors. N Engl J Med. 2015;372(26):2499-508.

[58] Diplas BH, He X, Brosnan-Cashman JA, Liu H, Chen LH, Wang Z, et al. The genomic landscape of TERT promoter wildtype-IDH wildtype glioblastoma. Nat Commun. 2018;9(1):2087.

[59] Arita H, Yamasaki K, Matsushita Y, Nakamura T, Shimokawa A, Takami H, et al. A combination of TERT promoter mutation and MGMT methylation status predicts clinically relevant subgroups of newly diagnosed glioblastomas. Acta Neuropathol Commun. 2016;4(1):79.

[60] Gilbert AR, Zaky W, Gokden M, Fuller CE, Ocal E, Leeds NE, et al. Extending the Neuroanatomic Territory of Diffuse Midline Glioma, K27M Mutant: Pineal Region Origin. Pediatr Neurosurg. 2018;53(1):59-63.

[61] Solomon DA, Wood MD, Tihan T, Bollen AW, Gupta N, Phillips JJ, et al. Diffuse Midline Gliomas with Histone
H3-K27M Mutation: A Series of 47 Cases Assessing the Spectrum of Morphologic Variation and Associated Genetic Alterations. Brain Pathol. 2016;26(5):569-80.

[62] Meyronet D, Esteban-Mader M, Bonnet C, Joly MO, Uro-Coste E, Amiel-Benouaich A, et al. Characteristics of H3 K27M-mutant gliomas in adults. Neuro Oncol. 2017;19(8):1127-34.

[63] Karremann M, Gielen GH, Hoffmann M, Wiese M, Colditz N, Warmuth-Metz M, et al. Diffuse high-grade gliomas with H3 K27M mutations carry a dismal prognosis independent of tumor location. Neuro Oncol. 2018;20(1):123-31.

[64] Ballester LY, Huse JT, Tang G, Fuller GN. Molecular classification of adult diffuse gliomas: conflicting IDH1/IDH2, ATRX, and $1 \mathrm{p} / 19 \mathrm{q}$ results. Hum Pathol. 2017;69:15-22.

\section{Address of correspondence:}

\section{Dr Debajyoti Chatterjee}

Assistant professor, Department of Histopathology

Room no 512, 5th floor, Department of Histopathology, Research block A, Postgraduate Institute of Medical Education and Research (PGIMER), Chandigarh, Pin-160012.

Email:devchat1984@gmail.com 